Article

\title{
3D Modelling for Solar Erythemal UV Protection Provided by Human Hair
}

\author{
Arianna Religi * and Laurent Moccozet $(1)$ \\ Centre Universitaire d'Informatique (CUI), University of Geneva, Route de Drize 7, 1227 Carouge, Switzerland; \\ laurent.moccozet@unige.ch \\ * Correspondence: arianna.religi@unige.ch
}

Received: 21 October 2019; Accepted: 4 November 2019; Published: 6 November 2019

check for updates

Featured Application: This research is applied to enable studies to be carried out on the exposure of the human body to UV radiation and its distribution over all parts of the body, head included, using a tool named SimUVEx. This study can provide preventive advice for the general public on how best to protect themselves when exposed to UV radiation, contributing to improve our understanding of exposure patterns, such as the shade, and identify some potential shortcomings in current sun protection recommendations.

\begin{abstract}
The head is one of the most sun-exposed anatomical zones and its ultraviolet (UV) exposure is often underestimated. The goal of this research is to quantify the solar erythemal UV protection provided by human hair for different orientations and Solar Zenith Angles (SZA). For this purpose, a three-dimensional (3D) numeric simulation tool called SimUVEx was used to forecast the distribution of UV exposure based on geometric information and solar irradiance. The relative UV dose reduction was expressed in terms of predictive protection factor (PPF). Results showed that the effectiveness of the protection depends strongly on the orientation of the head and on the tilt of the hairs. As the SZA increases, the PPF also increases. PPFs provided by hair with a certain degree of order (parallel alignment) were generally higher by a range of about $10 \%-30 \%$ than the ones provided by random arrangements. A significant exposure to the scalp through the hair, even for an average hair density, was found. It confirms that hair is an important natural sunscreen in reducing transmission of UV radiation and must be considered in the prevention messages of skin cancer on the scalp, particularly in people with alopecia.
\end{abstract}

Keywords: 3D human modelling; UV radiation; skin cancer; sun protection; human hair

\section{Introduction}

The skin is the most exposed organ to environmental ultraviolet (UV) radiation (280-400 nm), which is responsible for both beneficial and adverse effects on human health. An adequate exposure to solar radiation represents the main natural source of vitamin $\mathrm{D}$, the hormone that controls calcium levels in the blood and helps maintain healthy bones and muscles. On the contrary, prolonged exposures could give rise to severe and long-term effects on skin, eyes and immune system [1]. UV radiation has a significant influence on the development of cutaneous melanoma, epithelial skin cancer [2] and on the premature skin ageing [3]. Currently, 132,000 melanoma skin cancers and between 2 and 3 million non-melanoma skin cancers occur globally each year, with an average yearly increase of $3 \%-8 \%$ over the last 30 years among white populations in Australia, Europe, Canada and the United States [4-6]. It is not obvious to calculate the UV radiation exposure received throughout an entire life, since this would mean obtaining information on all the sun-exposure durations and geolocalisations. Moreover, the personal UV exposure depends on the distribution of the three components of the radiation (i.e., 
direct, diffuse and reflected) all over the human body and on behavioural factors such as the orientation to the sun, posture, skin complexion, garments and sun-protective habits [7-9]. Understanding the relationship between UV exposure and skin cancer on different anatomical zones is crucial then to define sun protection practices and messages [10-12]. Most of the existing measurements consist in photosensitive dosimeters and captors (e.g., [13-15]), which evaluate the integrated cumulative UV dose received at the specific location where they are affixed during the exposure. Despite their accuracy, dosimetry is expensive, and many dosimeters are needed to measure body-specific exposure, resulting in them being situation-specific and making generalization difficult. Though numerous studies examined the human body exposure to UV radiation [16,17], to our knowledge, little has been done about the application of human models and tools for predicting and visualizing UV exposure [18], often without taking into consideration sun protections. To address this issue, we developed SimUVEx (Simulating UV Exposure) [19,20], a modelling tool that combines three-dimensional (3D) techniques and ground-based UV irradiance to evaluate the relative contribution of the components of the UV radiation by different anatomical zones and in various exposure conditions. Typically, exposures of anatomical zones range from $13 \%$ to $76 \%$ of the exposure to the vertex of the head [21], and horizontal body parts exhibit higher exposure doses [22,23]. Except for a continued use of a headgear, the head is then one of the most sun-exposed anatomical parts, and the real-time exposure is often underestimated. Moreover, strong dissimilarities in the incidence of head and neck melanoma between men and women have been observed in previous researches [24], bringing to the conclusion that hair might have a protective role in sun exposure.

The goal of this paper is to improve our model so that it could estimate how much UV exposure can reach the epidermis of the head, covered by hair, without sun damage risks. The ease and the speed of the model were key points to take into consideration in numerous scenarios, while using a computer with standard requirements. The hairs were considered as independent 3D objects, with different alignments and orientations in relation with the solar position, that is, the solar zenith angle (SZA) that represents the angle between the zenith and the line of sight from that point to the sun. To the best of our knowledge, a very small number of studies have tried to quantify the effectiveness of the hair to protect against UV exposure, by spectrometric techniques [24] or dosimetries [25,26], but none of them used 3D modelling methods. Realistic hair modelling is very important for creating virtual humans in computer graphics, but studies are often limited to hairstyling and hair rendering, looking further into the effects of the visible light on the surface of the hair without taking into consideration other spectra, such as the UV wavelengths [27-30]. This study investigates the behaviour of the hairs in relation with their geometric distribution in the erythemally weighted UV spectrum, adopted by the International Commission on Illumination (Commission Internationale de $1^{\prime}$ Eclairage, CIE) and representing the average skin response over the solar UV spectrum [31].

\section{Materials and Methods}

SimUVEx is a modelling tool built on the Visualisation and Computer Graphics Library (VCG) [32] and implemented as a plug-in in Meshlab [33], an open-source system for computing and editing 3D triangular meshes. An overall description of the tool is represented in Figure 1. The principles, the performances and its validation have been described previously [19,20,34-37]. 


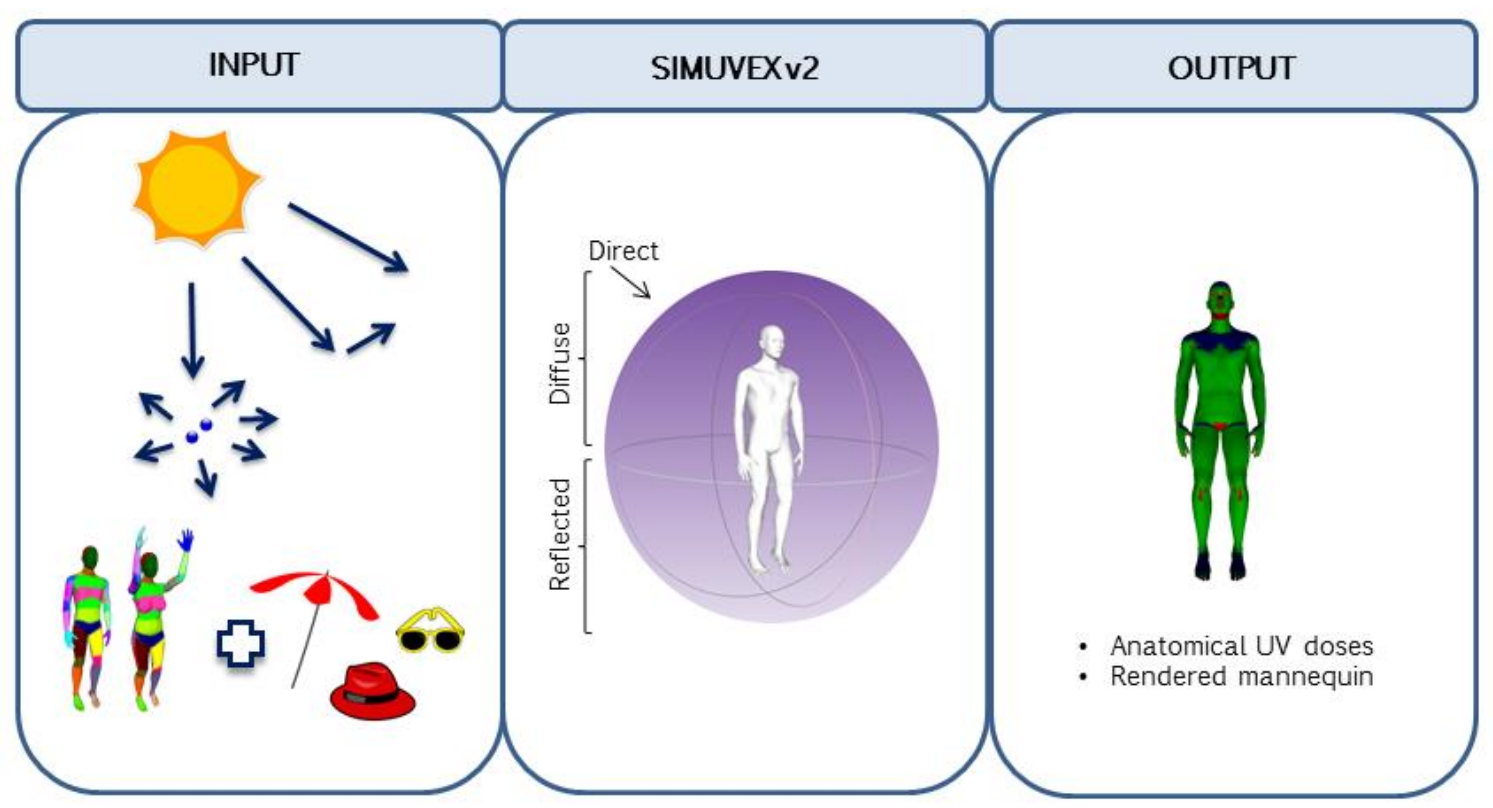

Figure 1. General overview of SimUVEx (Simulating UV Exposure). UV: ultraviolet.

Input. The requisite input data of the model are the sun position, the irradiance (in $\mathrm{W} / \mathrm{m}^{2}$ ) and the body morphologies and postures. Ground-based erythemally weighted UV irradiances have been collected by SolarLight SL 501A broadband radiometers at the MeteoSwiss Payerne Station $\left(46.815^{\circ} \mathrm{N}\right.$, $6.944^{\circ} \mathrm{E}$, altitude $491 \mathrm{~m}$ ) for the direct, diffuse and reflected components. The human body mannequins were designed with MakeHuman [38], a modelling tool for 3D human characters based on articulated skeleton techniques and on anatomical atlases [39,40], to create 3D surfaces of five morphologies (adult man and woman, adult heavy man, a child, and a head-form), shown in Figure 2.

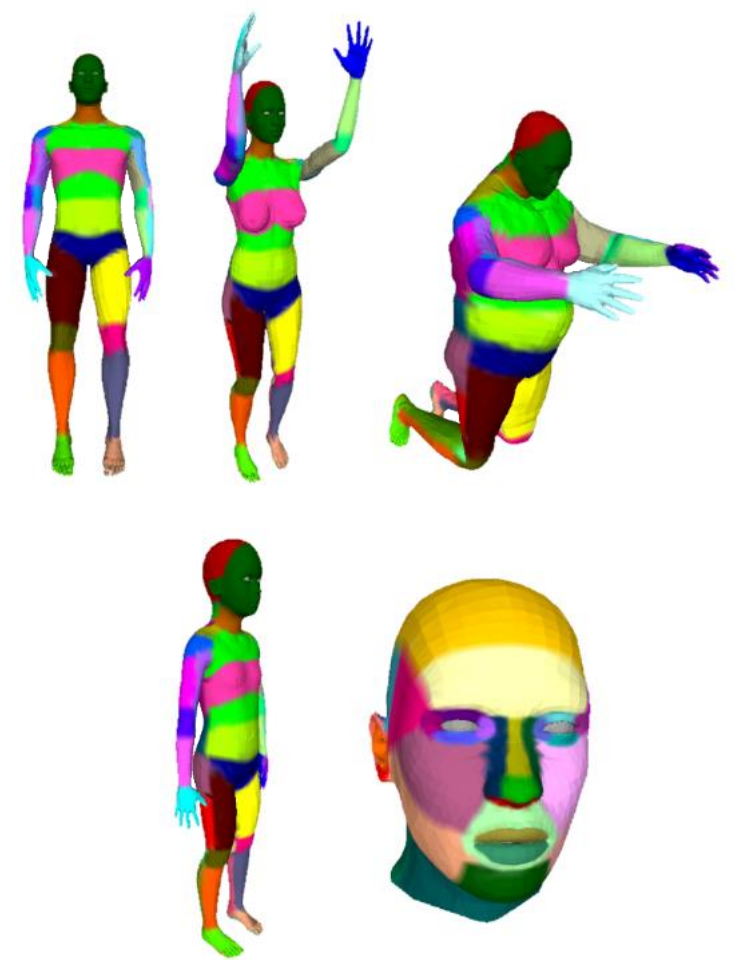

Figure 2. The five morphologies of SimUVEx: (on the top) adult man, adult woman, heavy man; (on the bottom) child, head-form. Each colour represents an anatomical zone. 
Depending on the size density of the 3D meshes, a low and a high resolution were provided for each morphology. For the low resolution, we used 800 vertices for the entire body, and 1800 vertices were used for the high resolution. Then, we used Blender [41], an open-source 3D computer graphics software for interactive applications, to modify the articulated skeleton and set different postures. Globally, six working postures were designed, based on the most predominant postures among outdoor workers [42,43], and three leisure time postures, based on the observation of the most common postures on the beaches. The duration of the simulation is determined by the resolution; on a standard desktop workstation it takes about 14 minutes for one day simulation ( $24 \mathrm{~h}$ ) in high-resolution mode and about one minute for the low resolution. Depending on the type of the simulation chosen, the position can either be fixed ("fixed mode") for every time iteration or it can change on a given time and angle step to obtain an average orientation ("sequence mode"), since people hardly stay static in everyday situations.

Each 3D mannequin is subdivided in various anatomical zones, represented by a different RGB colour, as shown in Figure 2. In total, 46 subanatomical zones were selected for the whole body (Table 1), whereas for the head, 36 zones (Table 2) were distinguished, according to the International Classification of Disease for Oncology [44]. We considered many anatomical zones defined by skin cancer registers to have the possibility to compare our results with the existing results from skin cancer research.

Table 1. Detailed anatomical regions and subregions for the human model in SimUVEx.

\begin{tabular}{ll}
\hline \multicolumn{1}{c}{ Anatomical Zone (Body) } & \multicolumn{1}{c}{ Subanatomical Zones } \\
\hline Head & Skull, Face \\
Neck & Front, Back \\
Trunk & Top, Front, Torso, Chest ${ }^{* *}$, Belly, Lower belly \\
Trunk Back & Upper, Lower, Posterior \\
Arm * & Shoulder, Upper arm inside, Upper arm outside \\
Elbow * & Inside, Outside \\
Lower Arm * & Inside, Outside \\
Hand * & Palm, Back \\
Leg * & Upper front, Upper back \\
Knee * & Front, Back \\
Lower Leg * & Front, Back \\
Foot * & Palm, Back \\
\hline & *for left and right side. \\
& ** available only for high resolution.
\end{tabular}

Table 2. Detailed anatomical regions and subregions for the facial region.

\begin{tabular}{ll}
\hline \multicolumn{1}{c}{ Facial Zone } & \multicolumn{1}{c}{ Subanatomical Zones } \\
\hline Head & Forehead, Crown, Nape \\
Face & Cheek , Mandible *, Chin, Temple* \\
Neck & Front, Back \\
Orbital region * & Tear-duct, Upper, Lower, Lateral \\
Internal eye* & Pupil, White* \\
Ears * & Auricula, Earlobe, Earlobule front/back \\
Nose & Columnella, External nose *, Tip, Dorum nasale \\
Oral region & Upper lip, Lower lip, Orbicularis oris \\
\hline & *for left and right side.
\end{tabular}

The model. The amount of UV irradiance is calculated considering the three radiation components and shading from other body parts for each triangular mesh. The direct radiation is considered a parallel source, varying in intensity and in direction. The diffuse and reflected components depend on the surface orientation and the ratio of the sky visible, not obstructed by the shade. In SimUVEx, the diffuse and reflected irradiances are considered hemispherical isotropic sources, meaning that an 
equal amount of energy is assigned to each subsurface element of the bottom half-sphere. In reality, since diffuse radiation is anisotropic, particularly near the ground and close to the sun during clear days [45], SimUVEx takes account of anisotropy near the ground, attenuating the energy allocated to subsurfaces at the base of the diffuse half-sphere. Then, a bounding box optimization has been applied to determine if a vertex was in the shade, and a "visibility map" has been conceived to detect the surfaces of each hemisphere visible from the vertex, for the diffuse and reflected radiation [46]. The implementation of visibility maps allows a whole variety of 3D sun protection means such as hats, sunglasses or shade structures [20,35-37], for whom the model can calculate their shade effects on the mannequin, similarly to the shadow of other body parts.

Output. The output of the model consists in the UV erythemal radiance (in $\mathrm{J} / \mathrm{m}^{2}$ ) one-minute resolution for each body part, whose result can be also retrieved on a 3D human mannequin, where the redder the anatomical zone, the more it is exposed.

Protection factors. The protection factor is an optional parameter that reduces the amount of UV radiation received by the covered vertices. Generally, it is associated to a homogenous value which depends on the textile or material of the garment, and the most used factors are the sun protection factor (SPF), the standard used to measure the effectiveness of sunscreens, and the ultraviolet protection factor (UPF), that is more recent and established specifically for sun-protective fabrics. SPF measures the amount of time it takes for sun-exposed skin to redden, considering only UV-B radiation (280-315 nm). For instance, if one burns in 10 minutes without sun protection and applies a sunscreen with $15 \mathrm{SPF}$, it should be protected from sunburn for 150 minutes. On the contrary, the UPF factor quantifies the amount of UV irradiance absorbed by the fabric. A material with an UPF equal to 50 allows 1/50th of the UV irradiance to pass through it. This means that the fabric will reduce skin UV exposure by 50 times in areas where the skin is covered. In light of the above, these factors do not clearly inform the public about the quantity of UV transmitted to the skin when using sun protections [47] and do not consider the diffuse UV radiation that has recently been shown to contribute substantially to the total UV exposure, a fact probably underrated in general prevention messages based on dosimetric measurements [23]. The protection factor in SimUVEx is defined as the ratio between the received erythemal UV irradiance without protection and the received erythemal UV irradiance after protection, likewise the UPF [25]. It is then different from the sun protection means (i.e., sunglasses, hats, shade structures) that are specific 3D meshes, for which the model calculates the shadow they produce on the mannequin throughout the whole simulation and for the three components.

Hair modelling as sun protection mean. Hair modelling is an intricate task, since a realistic representation in structure, motion, and appearance is still a purpose of research $[29,48]$. Moreover, the existing models relate to visible light, without considering UV radiation. Each hair strand is relatively small in diameter, while a human head typically contains a large volume of hair. Bearing in mind this duality, researchers have examined whether hair should be treated as an overall volume or as individual interacting hair strands, but neither of them have been accepted, and the choice lies on what aspect one should explore. For our purpose, we considered the hair as a combination of 3D objects to evaluate their effectiveness first. This parametric geometric approach has been used to investigate the shadow that each hair projects to the scalp, similarly to other protections means such as hats or sunglasses. Then, we considered SimUVEx as a virtual laboratory that could simulate samples of skin covered by hair. The results obtained allowed us to estimate various average protection factors that can be integrated in the protection libraries of SimUVEx.

The hair samples were designed with Blender as opaque objects, providing full shielding against $\mathrm{UV}$ radiation, and were devised as cylinder meshes of $3 \mathrm{~cm}$ in length, as shown in Figure 3. 


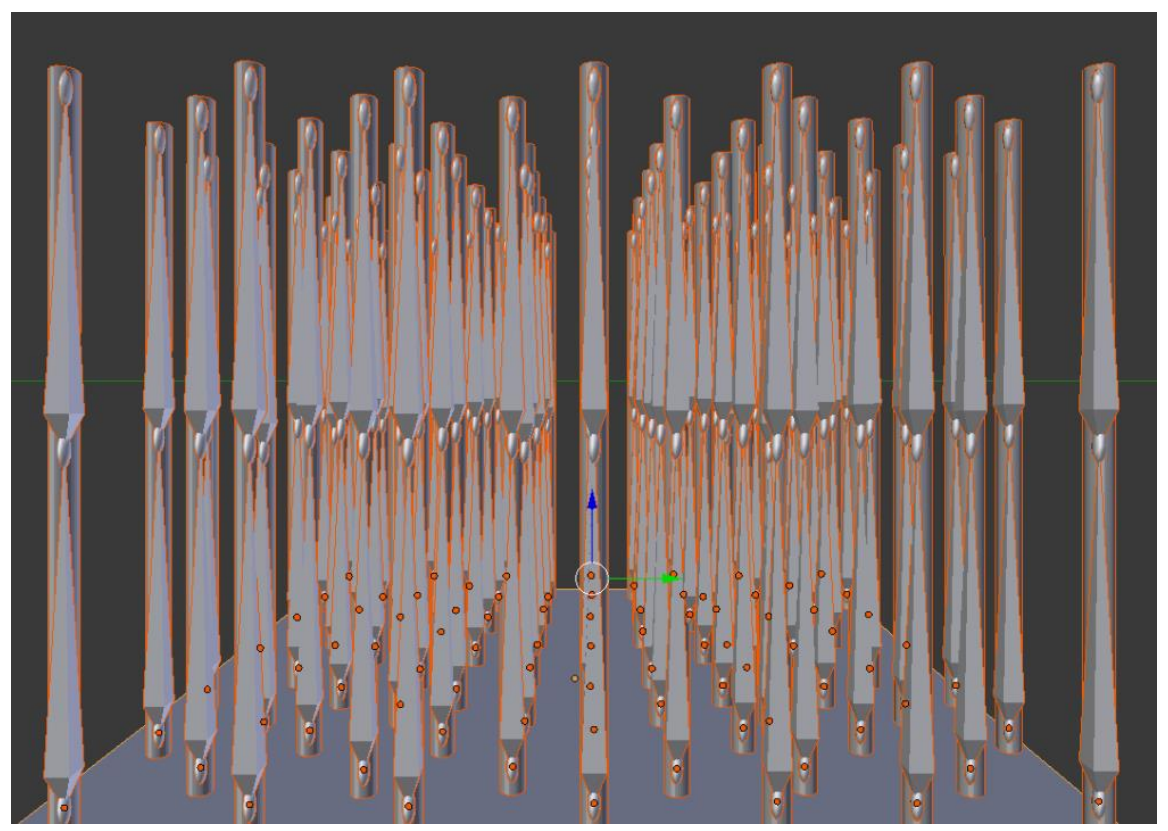

Figure 3. Hair surface showing two bones armature that supports the structure of the cylinder.

A two bones armature (skeleton), that is, the supporting structure of the 3D model represented in Figure 4, was implemented for each hair according to the rigging method. The armature was then connected to the mesh through the skinning process. The tilt of the hairs is a parameter that can be set by the user.

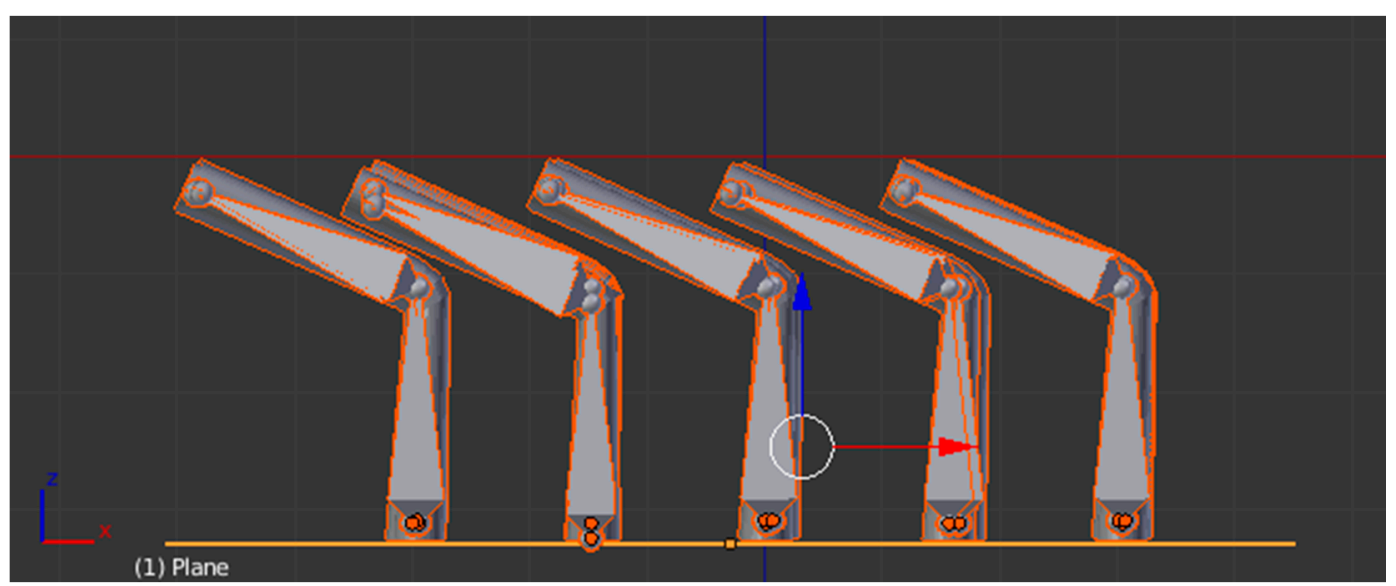

Figure 4. Example of the rigging effect for the middle bone: the tilt of the hairs is a parameter that can be adjust by the user.

We depicted an average scenario for a medium-low hair density (11-20 hairs in about $18 \mathrm{~mm}^{2}$ ) and a medium hair diameter (50-80 $\mu \mathrm{m})$, in accordance with [49]. To evaluate the degree of sunscreen provided by the structure of hair, we set four alignments on a squared surface of $1 \mathrm{~cm}^{2}$ : upright, random, parallel alignment (Figure 5). 


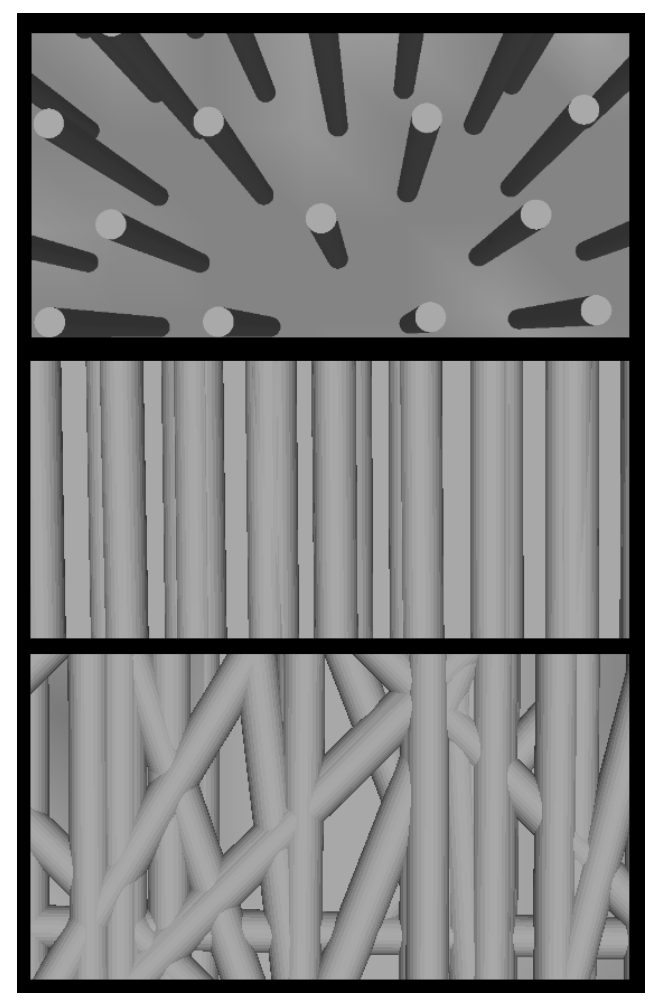

Figure 5. Upright/Straight/Random orientations of the hair (top view).

For the random and parallel alignment, hairs were tilted at $180^{\circ}$. With the aim to consider the influence of the SZA throughout the day and the different parts of the head (the crown, the nape and the lateral parts), we inclined the surface with respect to the sun in all the possible orientations: on the top, right, left, back, front. A dynamic exposure was presumed, selecting a $24^{\circ}$ step rotation per minute to consider an average value. Simulations were run at the Payerne MeteoSwiss Station for a full day (12AM-12PM) in a summer, cloudless day (17/07/2014), since the effect of clouds on instantaneous UV levels can vary from a small amplification to an almost total decrease [50].

For every skin region, the sun protection effectiveness was expressed as a predictive protection factor (PPF (\%)), representing the relative reduction in predicted total UV dose:

$$
\operatorname{PPF}[\%]=\frac{U V_{\text {without protection }}-U V_{\text {with protection }}}{U V_{\text {without protection }}} \times 100
$$

where $U V_{\text {without protection }}$ is the UV exposure received on uncovered skin and $U V_{\text {with protection }}$ is the exposure received on the skin covered by hair. The greater the PPF, the higher the relative sun dose reduction. All the PPF values refer to the total radiation since they represent the contribution of all the UV radiation components.

\section{Results}

The PPFs (\%) to the head through the hair for upright, random and parallel hairs are provided in Table 3 for different SZAs during a full clear-sky day. For each case, the surface was placed in four orientations (on the top, the front, the back, the right and the left). All the PPFs range from about 35\% to $80 \%$, with a general increase with the SZA. Indeed, the UV exposure through the hair decreases with the SZA, since UV radiation is stronger for lower SZAs (sun at its highest) compared with higher SZAs (lower sun), as the light travels through a shorter path of the atmosphere. 
Table 3. PPFs (\%) for different Solar Zenith Angles (SZAs). PPF: predictive protection factor.

\begin{tabular}{cccccc}
\hline & Top & Front & Back & Right & Left \\
\hline SZA & \multicolumn{5}{c}{ Upright } \\
\hline $25^{\circ}-30^{\circ}$ & 44.4 & 52.4 & 47.1 & 45.8 & 45.9 \\
$30^{\circ}-35^{\circ}$ & 47.0 & 51.7 & 47.1 & 45.8 & 47.4 \\
$35^{\circ}-40^{\circ}$ & 47.4 & 52.5 & 47.1 & 46.4 & 49.7 \\
$>40^{\circ}$ & 48.5 & 51.1 & 46.1 & 47.1 & 46.5 \\
\hline \multicolumn{5}{c}{ Parallel alignment } \\
\hline $25^{\circ}-30^{\circ}$ & 63.9 & 75.7 & 61.9 & 73.7 & 62.3 \\
$30^{\circ}-35^{\circ}$ & 58.0 & 68.4 & 61.9 & 74.2 & 56.7 \\
$35^{\circ}-40^{\circ}$ & 62.7 & 66.5 & 61.9 & 77.5 & 55.4 \\
$>40^{\circ}$ & 65.6 & 69.1 & 79.2 & 63.4 & 56.5 \\
\hline & \multicolumn{5}{c}{ Random } \\
\hline $25^{\circ}-30^{\circ}$ & 50.9 & 67.7 & 48.0 & 34.4 & 38.0 \\
$30^{\circ}-35^{\circ}$ & 51.2 & 52.5 & 48.0 & 34.2 & 30.9 \\
$35^{\circ}-40^{\circ}$ & 53.8 & 53.0 & 48.0 & 42.0 & 32.3 \\
$>40^{\circ}$ & 48.8 & 54.9 & 62.8 & 39.2 & 39.9 \\
\hline
\end{tabular}

Comparing all the alignments, higher PPFs were found in parallel-aligned hairs, which usually cover homogeneously a larger part of the scalp. While the PPFs for parallel alignment range from $55 \%$ to $80 \%$, upright alignment provides the lowest values (PPFs between $44 \%$ and $52 \%$ ) and consequently more exposure, as a greater surface area of the scalp is prone to UV irradiance intrusion. The random arrangement shows results halfway between the two alignments for the top, the front and the back orientations. On the contrary, it is less protective (from $30 \%$ to $42 \%$ ) for the right and left orientations of the head, even if the random alignment is less frequent in these parts.

The front shows a good protection (higher than $50 \%$ ) when the sun is at its highest $\left(25^{\circ}-30^{\circ}\right)$, since the hairs are perpendicular to the incoming light, offering more shadow. Values of the back are quite constant for all situations, except for higher SZAs, when the protection is stronger.

PPFs for each situation throughout the day are shown in Figure 6.

PPF is generally higher for the parallel alignment and smaller for upright arrangement, which lets more radiation pass through. Except for the back, each situation reveals a peak where the dose exposure increases and the sun is at its highest elevation, around 12:00, corresponding to a minimum SZA value. Upright alignment shows a constant behaviour for all situations throughout the day, with values between $40 \%$ and $50 \%$. The parallel and random alignments have a similar behaviour, with specular curves in the left and right cases. For the left and right side, the evaluation of the model is not trivial, as the results depend more significantly on the position of the sun, at both the zenithal and azimuthal levels. Therefore, it is not always easy to find a general explanation. In our case, we can observe that for the right and left sides, the random PPFs are once again smaller than that of upright alignment, in agreement with common sense, but in some cases, they are smaller than that of parallel alignment as well. This is probably due to the fact that upright alignment, in lateral zones, can provide more protection, since the hair strand is often perpendicular to UV rays, providing then more shade on the anatomical zone considered. However, upright alignment for right and left areas is more realistic for beard than for hair. On the contrary, a random behaviour, which is not in an upright position by definition, would protect less areas in terms of shade. 

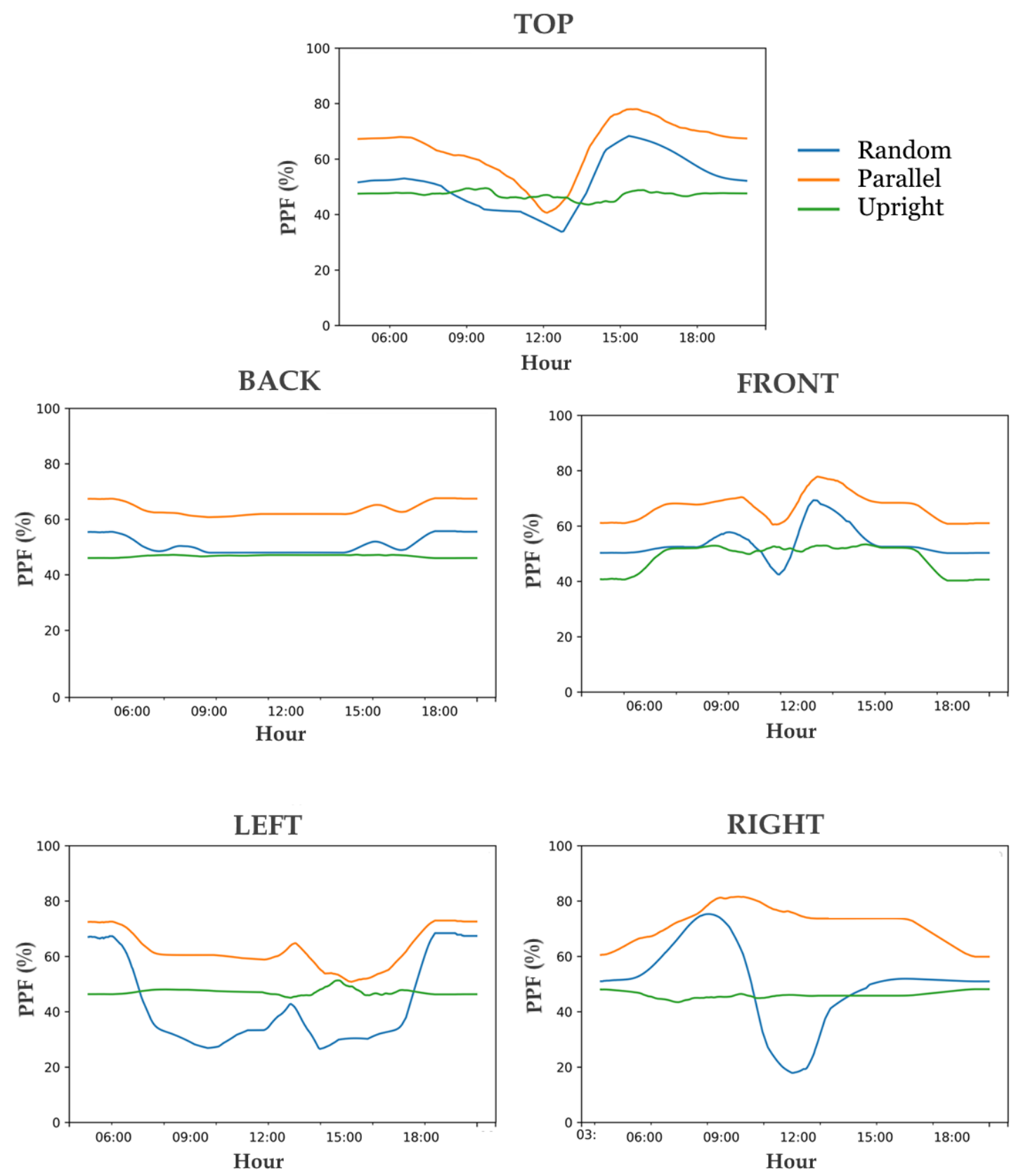

Figure 6. Daily PPFs using a moving average smoothed curve with 1 hour window for all the hair orientations and alignments. The marked hours in the plots $(6,9,12,15,18)$ correspond to the following SZA: $71.1^{\circ}, 40.9^{\circ}, 26^{\circ}, 47.9^{\circ}, 78.2^{\circ}$.

\section{Discussion}

The study quantifies the erythemal UV protection through human hair for different orientations and SZAs using SimUVEx, a 3D modelling tool predicting solar UV exposure. Simulations were run in a clear-sky day to eliminate cloudy situations that can affect significantly UV exposure. The hairs were considered opaque objects, providing full shielding against UV radiation. Different scenarios have been conceived according to the fact that UV protection provided by hair may be influenced by the density, the thickness, the colour, the alignment and the tilt of the head with respect to the sun. In this research, the density and thickness were fixed, and we focused on the geometric influence of hair protection over the head, studying specifically the effects of the alignment and the tilt of the hair. We depicted an average scenario, taking into consideration a medium-low hair density and a medium hair 
diameter $(50-80 \mu \mathrm{m})$ [49] for hairs approximately $3 \mathrm{~cm}$ in length. We chose to investigate short hair effects to better understand the link between orientation of the hair and position of the sun. Indeed, the case of long hair would have been more protective, since it would have covered almost the entire scalp surface. We did not consider the dynamic motion of hair because the movements of the head and the body do not lead to big change in the short hair, so that the model can consider the hair as a static object. Low-medium hair density was chosen to investigate the most endangered cases among men. We did not treat the influence of colour in this analysis since it can be considered as an extra protection factor, as well as the skin phototype. Indeed, hair colour results from the biosynthesis of melanin within hair follicles. Melanin molecules in the skin constitute a built-in defence system to protect the skin against UV radiation, and when a follicle stops producing melanin, the hair turns colourless [51]. A dark-skinned person has more melanin molecules in the upper layers of the skin than a light-skinned person [52]. According to [24], approximately 300 hairs $\mathrm{cm}^{-3}$ would be needed to achieve an SPF of 0-10 in the case of brown, blond and red hair, and about 400 hairs $\mathrm{cm}^{-3}$ of white hair. Moreover, it is noteworthy that SPF ratings are calculated for an application thickness of $2 \mathrm{mg} \mathrm{cm}^{-2}$, which is unrealistic, as most people apply significantly thinner layers. Additionally, results for bald situations can be measured in the usual routine of SimUVEx.

Protection was estimated in terms of PPF that represents a sort of mean bias that is the difference between the values with and without hair protection. It does not indicate the amount of UV reaching the skin, but just the effectiveness of the protection.

As the SZA increases, the PPF also increases for all hair types. PPFs provided by hair with a certain degree of order (parallel alignment) were generally higher by a range of about $10 \%-30 \%$ than the ones provided by random arrangements. This is due to the fact that tidy arrangements may confer higher cover than random alignments because of the loss of effectiveness by overlap of hair [25]. On the contrary, upright orientation provided more exposure because a greater surface area of the scalp is prone to solar radiation intrusion, especially at around noon (minimum SZA).

Considerable progresses have been made in the understanding of artificial sun protections such as hats [20,37,53], sunglasses [36] or shade structures [35,53-56], but little is still known about the photo-protective function of human hair. The effects of UV radiation on hair have only lately attracted the attention of the medical community. The incidence of melanoma is rising, and it is evident that melanoma is less common in hair-covered areas of the head and the neck compared with naked areas, suggesting a protective role for hair [57]. Moreover, vacations in sunny areas and outdoor activities are growing in popularity, making more and more important the awareness of sun protection on the skin. The two most important chronic effects of UV radiation on the skin and bare scalp are photocarcinogesis and solar elastosis [58]. The relativity high incidence of precancerous lesions and skin cancers in this area [59] could be explained by the fact that a person standing, has always his scalp perpendicular to the sun's rays and therefore receives high UV doses. Even if the estimation of UV erythemal radiation absorbed by the head was not the focus of the study, this research showed that there may be considerable exposures to the scalp through the hair, even for an average hair density (PPFs are never close to $100 \%$ ). It confirms that hair is an important natural sun protection means in reducing transmission of $U V$ radiation and should be considered in the prevention of UV-induced cancer of the scalp, particularly among people with alopecia. The effectiveness of the protection depends strongly on the orientation of the head and on the tilt of the hairs and was in line with other epidemiological results [24-26].

The results obtained will be implemented in the library of the sun protection factors of SimUVEx, assessing a more exhaustive model that could include the hair protection, which is not a negligible factor in terms of UV exposure. In this way, it could be possible to set more realistic scenarios and obtain an innovative tool in estimating effective sun prevention for further simulations, especially for the detailed head form morphology, instead of considering the bald head used so far in the model. Future works could also be focused on the estimation of the UV radiation intercepted by hairs themselves to further quantify the hair colour changes and protein damage caused by ultraviolet radiation $[60,61]$. 
Another interesting scenario from a medical point of view could be to consider new hair densities (lower or higher) and lengths (shorter and longer).

Author Contributions: A.R. and L.M. conceived of the presented idea. L.M. designed SimUVEx, A.R. developed the 3D models and performed the analytical computations. Then L.M. analysed and discussed the results with A.R.

Funding: This research was funded by the Swiss National Science Foundation, grant number CR2313 152803, and is entitled PuRSUE (Predict Solar UV Exposure, full title: Ground UV irradiance and 3D rendering techniques to predict anatomical solar UV exposure in Skin cancer research).

Acknowledgments: We would like to express our gratitude to Laurent Vuilleumier for support and allowing us to work on MeteoSwiss data collected at the Payerne Meteorological Station (Switzerland). We also thank David Vernez, Jean-Luc Bulliard and Claudine Backes for their insightful comments throughout the project.

Conflicts of Interest: The authors declare no conflict of interest.

\section{References}

1. Vainio, H.; Wilbourn, J. Identification of carcinogens within the IARC monograph program. Scand. J. Work Environ. Health 1992, 18, 64-73.

2. Blum, A.; Volkenandt, M.H. Skin cancer. DMW Dtsch. Med. Wochenschr. 2002, 127, 1679-1681. [CrossRef]

3. Fisher, G.J.; Wang, Z.Q.; Datta, S.C. Pathophysiology of premature skin aging induced by ultraviolet light. N. Engl. J. Med. 1997, 337, 1419-1429. [CrossRef]

4. Rogers, H.W.; Weinstock, M.A.; Harris, A.R. Incidence estimate of nonmelanoma skin cancer in the United States, 2006. Arch. Dermatol. 2010, 146, 283-287. [CrossRef]

5. Fransen, M.; Karahalios, A.; Sharma, N. Non-melanoma skin cancer in Australia. Med. J. Aust. 2012, 197, 565-568. [CrossRef]

6. Surdu, S.; Fitzgerald, E.F.; Bloom, M.S. Occupational exposure to ultraviolet radiation and risk of non-melanoma skin cancer in a multinational European study. PLoS ONE 2013, 8, e62359. [CrossRef]

7. Hill, D.; White, V.; Marks, R. Melanoma prevention: Behavioral and nonbehavioral factors in sunburn among an Australian urban population. Prev. Med. 1992, 21, 654-669. [CrossRef]

8. Parisi, A.V.; Kimlin, M.G.; Lester, R. Lower body anatomical distribution of solar ultraviolet radiation on the human form in standing and sitting postures. J. Photochem. Photobiol. B Biol. 2003, 69, 1-6. [CrossRef]

9. Autier, P.; Boniol, M.; Doré, J.F. Sunscreen use and increased duration of intentional sun exposure: Still a burning issue. Int. J. Cancer 2007, 121, 1-5. [CrossRef] [PubMed]

10. Stanton, W.R.; Janda, M.; Baade, P.D. Primary prevention of skin cancer: A review of sun protection in Australia and internationally. Health Promot. Int. 2004, 19, 369-378. [CrossRef]

11. Glanz, K.; Buller, D.B.; Saraiya, M. Reducing ultraviolet radiation exposure among outdoor workers: State of the evidence and recommendations. Environ. Health 2007, 6, 22. [CrossRef] [PubMed]

12. World Health Organization. Cancer Control: Knowledge into Action. WHO Guide for Effective Programmes: Prevention; World Health Organization: Geneva, Switzerland, 2007.

13. Turnbull, D.J.; Parisi, A.V. Dosimeter for the measurement of UV exposures related to melanoma induction. Phys. Med. Biol. 2010, 55, 3767. [CrossRef] [PubMed]

14. Weihs, P.; Schmalwieser, A.; Reinisch, C. Measurements of personal UV exposure on different parts of the body during various activities. Photochem. Photobiol. 2013, 89, 1004-1007. [CrossRef] [PubMed]

15. Siani, A.M.; Casale, G.R.; Modesti, S. Investigation on the capability of polysulphone for measuring biologically effective solar UV exposures. Photochem. Photobiol. Sci. 2014, 13, 521-530. [CrossRef] [PubMed]

16. Kimlin, M.G.; Parisi, A.V.; Downs, N.D. Human UVA exposures estimated from ambient UVA measurements. Photochem. Photobiol. Sci. 2003, 2, 365-369. [CrossRef]

17. Hoeppe, P.; Oppenrieder, A.; Erianto, C. Visualization of UV exposure of the human body based on data from a scanning UV-measuring system. Int. J. Biometeorol. 2004, 49, 18-25. [CrossRef]

18. Streicher, J.J.; Culverhouse, W.C., Jr.; Dulberg, M.S. Modeling the Anatomical Distribution of Sunlight. Photochem. Photobiol. 2004, 79, 40-47.

19. Religi, A.; Moccozet, L.; Farahmand, M. Simuvex v2: A numeric model to predict anatomical solar ultraviolet exposure. In Proceedings of the 2016 SAI Computing Conference (SAI), London, UK, 13-15 July 2016; pp. 1344-1348. 
20. Religi, A.; Moccozet, L.; Vernez, D. Prediction of anatomical exposure to solar UV: A case study for the head using SimUVEx v2. In Proceedings of the 2016 IEEE 18th International Conference on E-Health Networking, Applications and Services (Healthcom), Munich, Germany, 14-16 September 2016; pp. 1-6.

21. Diab, R.; Wright, C.; Martincigh, B. Anatomical distribution of ultraviolet solar radiation. S. Afr. J. Sci. 2004, 100, 498-500.

22. Diffey, B.L. An overview analysis of the time people spend outdoors. Br. J. Dermatol. 2011, 164, 848-854. [CrossRef]

23. Vernez, D.; Milon, A.; Vuilleumier, L. Anatomical exposure patterns of skin to sunlight: Relative contributions of direct, diffuse and reflected ultraviolet radiation. Br. J. Dermatol. 2012, 167, 383-390. [CrossRef]

24. De Gàlvez, M.V.; Aguilera, J.; Bernabò, J.L. Human hair as a natural sun protection agent: A quantitative study. Photochem. Photobiol. 2015, 91, 966-970. [CrossRef] [PubMed]

25. Parisi Alfio, V.; Smith, D.; Schouten, P. Solar ultraviolet protection provided by human head hair. Photochem. Photobiol. 2009, 85, 250-254. [CrossRef] [PubMed]

26. Parisi, A.V.; Turnbull, D.J.; Downs, N. Dosimetric investigation of the solar erythemal UV radiation protection provided by beards and moustaches. Radiat. Prot. Dosim. 2011, 150, 278-282. [CrossRef] [PubMed]

27. Khouas, L.; Odet, C.; Friboulet, D. 3D furlike texture generation by a 2D autoregressive synthesis. J WSCG 1998, 6, 171-177.

28. Yacoob, Y.; Davis, L.S. Detection and analysis of hair. IEEE Trans. Pattern Analysis Mach. Intell. 2006, 28, 1164-1169. [CrossRef] [PubMed]

29. Ward, K.; Bertails, F.; Kim, T.Y. A survey on hair modeling: Styling, simulation, and rendering. IEEE Trans. Vis. Comput. Graph. 2007, 13, 213-234. [CrossRef]

30. Selle, A.; Lentine, M.; Fedkwin, R. A mass spring model for hair simulation. ACM Trans. Graph. TOG. 2008, $27,64$.

31. McKenzie, R.; Blumthaler, M.; Diaz, S. Rationalizing nomenclature for UV doses and effects on humans. CIE WMO GAW Joint Rep. 2014, 211, 1-22.

32. Visualization and Computer Graphics Library (VCG). Available online: http://vcg.isti.cnr.it/vcglib/ (accessed on 5 November 2019).

33. Meshlab. Available online: www.meshlab.net (accessed on 3 October 2019).

34. Vernez, D.; Milon, A.; Francioli, L. A numeric model to simulate solar individual ultraviolet exposure. Photochem. Photobiol. 2011, 87, 721-728. [CrossRef]

35. Religi, A.; Backes, C.; Moccozet, L. Body anatomical UV protection predicted by shade structures: A modeling study. Photochem. Photobiol. 2018, 94, 1289-1296. [CrossRef]

36. Backes, C.; Religi, A.; Moccozet, L. Sun exposure to the eyes: Predicted UV protection effectiveness of various sunglasses. J. Expo. Sci. Environ. Epidemiol. 2019, 29, 753-764. [CrossRef] [PubMed]

37. Backes, C.; Religi, A.; Moccozet, L. Facial exposure to ultraviolet radiation: Predicted sun protection effectiveness of various hat styles. Photodermatol. Photoimmunol. Photomed. 2018, 34, 330-337. [CrossRef] [PubMed]

38. MakeHuman. Available online: http://www.makehumancommunity.org/ (accessed on 3 October 2019).

39. Di Giacomo, T.; Moccozet, L.; Magnenat-Thalmann, N. Towards Automatic Character Skeletonization and Interactive Skin Deformation. Eurograph. Stars 2007, 1, 47-61.

40. Di Giacomo, T.; Kim, H.; Moccozet, L.; Magnenat-Thalmann, N. Control structure and multi-resolution techniques for virtual human representation. In Shape Analysis and Structuring; Springer: Berlin/Heidelberg, Germany, 2008; pp. 241-274.

41. Blender. Available online: https://www.blender.org/ (accessed on 3 October 2019).

42. Milon, A.; Sottas, P.-E.; Bulliard, J.-L. Effective exposure to solar UV in building workers: Influence of local and individual factors. J. Expo. Sci. Environ. Epidemiol. 2007, 17, 58. [CrossRef]

43. Milon, A.; Bulliard, J.-L.; Vuilleumier, L. Estimating the contribution of occupational solar ultraviolet exposure to skin cancer. Br. J. Dermatol. 2014, 170, 157-164. [CrossRef]

44. Fritz, A.G. International Classification of Diseases for Oncology: ICD-O; World Health Organization: Geneva, Switzerland, 2013.

45. Kuchinke, C.; Nunez, M. An anisotropy correction method for all-sky measurements of diffuse UV-B erythemal irradiance. J. Atmos. Ocean. Technol. 2003, 20, 1523-1533. [CrossRef]

46. Bittner, J.; Wonka, P. Visibility in computer graphics. Environ. Plan. B Plan. Des. 2003, 30, 729-755. [CrossRef] 
47. Reinau, D.; Weiss, M.; Meier, C.R. Outdoor workers' sun-related knowledge, attitudes and protective behaviours: A systematic review of cross-sectional and interventional studies. Br. J. Dermatol. 2013, 168, 928-940. [CrossRef]

48. Bao, Y.; Qi, Y. A survey of image-based techniques for hair modeling. IEEE Access 2018, 6, 18670-18684. [CrossRef]

49. De Lacharriére, O.; Deloche, C.; Misciali, C. Hair diameter diversity: A clinical sign reflecting the follicle miniaturization. Arch. Dermatol. 2001, 137, 641-646.

50. Matthijsen, J.; Slaper, H.; Reinen, H.A.J.M. Reduction of solar UV by clouds: A comparison between satellite-derived cloud effects and ground-based radiation measurements. J. Geophys. Res. Atmos. 2000, 105, 5069-5080. [CrossRef]

51. Stoker, H.S. General, Organic, and Biological Chemistry; Nelson Education: Toronto, ON, Canada, 2012.

52. Slominski, A.; Wortsman, J.; Plonka, P.M. Hair follicle pigmentation. J. Investig. Dermatol. 2005, 124, $13-21$. [CrossRef] [PubMed]

53. Diffey, B.L.; Cheeseman, J. Sun protection with hats. Br. J. Dermatol. 1992, 127, 10-12. [CrossRef] [PubMed]

54. Turnbull, D.J.; Parisi, A.V.; Sabburg, J. Scattered UV Beneath Public Shade Structures During Winter. Photochem. Photobiol. 2003, 78, 180-183. [CrossRef]

55. Turnbull, D.J.; Parisi, A.V. Increasing the ultraviolet protection provided by shade structures. J. Photochem. Photobiol. B Biol. 2005, 78, 61-67. [CrossRef]

56. Utrillas, M.P.; Martinez-Lozano, J.A.; Nunez, M. Ultraviolet radiation protection by a beach umbrella. Photochem. Photobiol. 2010, 86, 449-456. [CrossRef]

57. Lesage, C.; Barbe, C.; Le Clainche, A. Sex-related location of head and neck melanoma strongly argues for a major role of sun exposure in cars and photoprotection by hair. J. Investig. Dermatol. 2013, 133, 1205-1211. [CrossRef]

58. Trüeb, R.M. Effect of ultraviolet radiation, smoking and nutrition on hair. In Alopecias-Practical Evaluation and Management; Karger Publishers: Basel, Switzerland, 2015; pp. 107-120.

59. Youl, P.H.; Janda, M.; Aitken, J.F. Body-site distribution of skin cancer, pre-malignant and common benign pigmented lesions excised in general practice. Br. J. Dermatol. 2011, 165, 35-43. [CrossRef]

60. Nogueira, A.C.S.; Joekes, I. Hair color changes and protein damage caused by ultraviolet radiation. J. Photochem. Photobiol. B Biol. 2004, 74, 109-117. [CrossRef]

61. Šebetic, K.; Sjerobabski, M.I.; Čavka, V. UV damage of the hair. Coll. Antropol. 2008, 32, 163-165. 